\title{
Research Article \\ Effectiveness of Oseltamivir Prophylaxis in Influenza Outbreaks in Residential Aged Care
}

\author{
Mithilesh Dronavalli ${ }^{1}$, Heidi Lord ${ }^{2,3}$, Kate Alexander ${ }^{1}$, Leng Boonwaat ${ }^{1}$, Narugopal Pal ${ }^{1}$, \\ Stephanie Mahalia Fletcher-Lartey ${ }^{1, *}$ \\ ${ }^{1}$ Public Health Unit, South Western Sydney Local Health District, Liverpool, New South Wales, Australia \\ ${ }^{2}$ Centre for Research in Nursing and Health, St George Hospital, South Eastern Sydney Local Health District, Australia \\ ${ }^{3}$ Centre for Evidence Based Initiatives in Health Care: A JBI Centre for Excellence, Wollongong, NSW, Australia
}

\section{ARTICLE INFO}

\section{Article History}

Received 17 October 2019

Accepted 20 March 2020

\section{Keywords}

Influenza

outbreaks

aged care facilities

vaccination

oseltamivir

prophylaxis

public health preparedness

\begin{abstract}
Influenza outbreaks in Aged Care Facilities (ACFs) can lead to hospitalizations and deaths. Influenza can spread rapidly through ACFs if precautionary measures are not taken. Along with influenza vaccination and precautionary hygiene measures, Oseltamivir Prophylaxis (OP) may be effective in reducing the attack rate of influenza by preventing new cases. A cohort study was carried out to investigate the effectiveness of OP use during influenza outbreaks in ACFs located within South Western Sydney Local Health District from 1 January 2015 to 31 December 2018. The main outcome assessed was the rate of OP failure (new cases of influenza in patients treated with OP) among ACF residents. Subgroups and various predictors of OP failure were investigated including presence of a dementia ward, high care ward, and days to Public Health Unit (PHU) notification. The cohort consisted of 86 ACF outbreaks involving 10,064 residents. OP prevented $90 \%$ of influenza cases during influenza outbreaks [0.1 RR (95\% confidence interval (CI): 0.08-0.12); $P<0.0001]$. ACFs with dementia wards had a $44 \%$ ( 0.56 relative risk (RR) (95\% CI: 0.34-0.93); $P<0.05)$ lower OP failure rate. ACFs with high level care had an $87 \%(0.13$ RR (95\% CI: 0.05-0.38); $P<0.05)$ lower OP failure rate. OP is highly effective in preventing new cases of influenza during outbreaks in ACFs, especially in ACFs with dementia or high care wards. Mandatory reporting of influenza outbreaks to PHUs would ensure that ACFs are supported throughout the outbreak, which will facilitate reductions in hospitalizations and mortality.
\end{abstract}

(C) 2020 South Western Sydney Local Health District. Published by Atlantis Press International B.V. This is an open access article distributed under the CC BY-NC 4.0 license (http://creativecommons.org/licenses/by-nc/4.0/).

\section{INTRODUCTION}

Influenza is a respiratory virus that can lead to deaths and hospitalizations in the elderly, especially during influenza outbreaks in Aged Care Facilities (ACFs) [1]. Residents in ACFs are particularly vulnerable to influenza given their age (over 65 years) and multiple comorbidities including impaired oral intake, limited dexterity, and altered consciousness [1]. Contributing to the transmission of influenza within ACFs is the closed environments that they live in, where they have contact with other residents and multiple carers, which predisposes to rapid viral transmission.

Influenza is a laboratory-notifiable condition for which surveillance data is routinely collected in New South Wales (NSW), Australia [2]. However, it must be noted that while influenza is notifiable, influenza outbreak reporting by ACFs is not mandatory, but highly recommended by NSW Health. ACFs are encouraged by NSW Public Health Units (PHUs) to implement timely sourcing of antiviral medications for both influenza treatment and prophylaxis. In addition, PHUs provide ACFs with advice and support to implement other outbreak control measures [3]. In the absence of

"Corresponding author. Email: Stephanie.Fletcher@health.nsw.gov.au

Data availability statement: The data that support the findings of this study are available from the corresponding author [SF-L], upon reasonable request. early reporting and prevention strategies, influenza spreads rapidly throughout ACFs causing high attack rates. The window of opportunity to implement control strategies is often variable; and early detection and management of influenza in ACFs enables a prompt public health response.

In Australia, the cornerstone of preventive measures for influenza in ACFs includes promotion of influenza vaccination, early diagnosis, treatment, and implementation of infection control measures $[4,5]$. Annual influenza vaccination for ACF staff and residents is one of the main preventive measures adopted; however, vaccine efficacy among residents in ACFs is limited as this population group has a poor immune response to vaccination [6,7]. Staff vaccination is not mandated by law and very few ACFs in NSW have policies and procedures to enforce staff compliance with seasonal influenza vaccination.

The use of antiviral agents active against influenza A and B, such as oseltamivir, are currently available in Australia to use for treatment and prophylaxis in ACFs. Oseltamivir is an oral neuraminidase inhibitor that works through viral aggregation at the host cell surface (influenza A and B viruses), preventing newly formed copies of the virus from invading the noninfected cells. This prevents viral replication $[8,9]$. Treatment with oseltamivir has been shown to reduce the severity of influenza [10]; however, research evaluating 
influenza control measures are inconclusive on the effectiveness of Oseltamivir Prophylaxis (OP) for preventing the transmission of influenza in ACFs, with limited Randomized Controlled Trials (RCTs) conducted [11]. There are gaps in the literature on OP being a predictor of outbreak duration, attack rates, and rates of OP failure. An RCT conducted in the Netherlands using oseltamivir for prophylaxis compared with placebo concluded no benefit to oseltamivir, although this study was underpowered [12]. Another RCT conducted in Australia demonstrated that OP was associated with a reduction in attack rate, however, was not significant at reducing hospitalizations or mortality [13].

The local PHU policy for the recommended prescription of OP is generally for those selected residents on the same wing, floor, or ward as the influenza affected residents. However, this can vary depending on the layout of the facilities. In small ACFs, OP may be administered to all residents in the ACFs that are not already symptomatic.

The purpose of this study was to investigate the effectiveness of OP in influenza outbreak control for ACFs in a metropolitan Local Health District in Sydney, NSW, Australia. The effectiveness of OP was assessed in subgroups alongside other predictors of attack rates, hospitalization, death, oseltamivir usage (treatment and prophylaxis), and spread and duration of outbreaks. Approval was obtained from the South Western Sydney Local Health District (SWSLHD) Human Research Ethics Committee (LNR/15/ LPOOL/291) to conduct enhanced surveillance of reported influenza outbreaks in ACFs within SWSLHD.

\section{MATERIALS AND METHODS}

\subsection{Study Design}

This large cohort study used prospective administrative data from the Notifiable Conditions Information Management System on influenza outbreaks that occurred in ACFs located within SWSLHD between 1 January 2015 and 31 December 2018. All ACFs that reported influenza outbreaks during the study period were included in the study.

\subsection{Data Collection}

Data collected during the study period was part of routine outbreak and surveillance data collection for the PHU. Data routinely collected included (1) number of cases of influenza; (2) number of cases with Influenza-like Illness (ILI); (3) number of cases tested; (4) number of wings/sections of the facility; (5) number of residents in facility; (6) number of staff in facility; (7) number of wings/ sections affected; (8) onset of first and last case; (9) date outbreak notified to the PHU; (10) number of vaccinated residents and staff; (11) preventive measures implemented; (12) number of staff or residents hospitalized with ILI; (13) number of deaths among residents during the outbreak; (14) date when treatment and/or prophylaxis with oseltamivir commenced and ceased; (15) dementia wing or high or low care facility; and (16) if prophylaxis ceased and the reason why. Individual and facility level data were also available for the use of oseltamivir as treatment or prophylaxis in residents and staff for each ACF, in addition to ILI clinical case status and laboratory-confirmed influenza case status. All ACFs collected respiratory swabs to test for influenza. ACFs were contacted by the PHU to ensure completeness of data for each outbreak.

A confirmed case was defined as influenza diagnosed in an ACF resident by Polymerase Chain Reaction (PCR) by a laboratory.

The clinical case definition for an ILI was fever $\left(>38^{\circ} \mathrm{C}\right.$ or a history of fever) and cough or sore throat, in the absence of any other explanation for symptoms, consistent with the NSW Health influenza control guideline [14].

\subsection{Statistical Analysis}

The main outcome investigated by this study was OP failure in the residential care setting. Other outcomes assessed were attack rates (both laboratory and clinical), oseltamivir treatment and prophylaxis rate, death and hospitalization rates, outbreak duration, and degree of spread across the ACF. For the purposes of this study, outbreak duration was defined as the number of days from the onset of the first case to the date of the last case.

Oseltamivir prophylaxis failure was defined as new clinical cases of influenza that occurred in residents on OP. OP failure was calculated as the attack rate in residents who took OP divided by the attack rate of residents who did not take OP. The OP usage or non-usage rates were calculated at the facility level.

The outcome predictors assessed in this study include resident and staff vaccination rates, the presence of a dementia ward, high or low care setting of the ACF, number of days to PHU notification, and rates of use of oseltamivir as prophylaxis.

Baseline descriptive statistics, including means and proportions, were calculated for all variables. The crude relative risk for the effectiveness of $\mathrm{OP}$ was calculated by pooling the data, with the exposure defined as cases on OP and outcome defined as a case of OP failure.

Count data for cases, hospitalizations, deaths, and vaccinations were recorded at the ACF level. The denominator for rates was the total ACF resident population. Therefore, further statistical modelling was done using Poisson regression that uses rates as an outcome measure, where the effect size is the incident rate ratio. The linear regression coefficient was used when the outcome was the duration of the outbreak in days. Effect sizes and 95\% Confidence Intervals (CIs) were calculated for univariate analyses between predictors and outcomes. Stata v15.0 (StataCorp. LLC., 2017, Stata Statistical Software: Release 15. College Station, TX) was used for carrying out all analyses. Missing data were analyzed using casewise deletion.

\section{RESULTS}

A total of 86 influenza outbreaks in 49 ACFs in South Western Sydney were reported to the SWSLHD PHU during the study period of 1 January 2015 to 31 December 2018 involving 10,064 residents. All outbreaks in the study period were investigated by public health staff and data reviewed to ensure completeness.

Two of the 86 outbreaks investigated did not report data on OP failure, which is relevant to the primary hypothesis. Staff vaccination rates were not available for 27 outbreaks and 6 outbreaks did not report resident vaccination rates. 


\subsection{Baseline Data}

Influenza-like Illness was diagnosed in $16 \%$ of ACF residents, with an overall $9 \%$ of cases confirmed by PCR. The mean vaccination rates for ACF residents were $88 \pm 0.18 \%$ (range $0.01-1.0$ ) and for staff were $37 \pm 24 \%$ (range $0.6-100 \%$ ). The rate of prescribing oseltamivir for prophylaxis was $54 \%$ compared with $8 \%$ prescribed for treatment. Average OP failure cases were 1\% (range 0-10.4\%). Hospitalization rates were $2 \%$ (range $0-10.2 \%$ ), with deaths ranging from $0 \%$ to $3.5 \%$ of residents affected. The average number of residents in an ACF was 117 (range 14-476). The mean outbreak duration was 6.5 days (range 0-23 days) from the onset of the first case and the PHU was notified on average within 5.4 days (range $1-19$ days) (Table 1 ).

\subsection{Attack Rate}

In the cohort of 10,064 residents, the attack rate in ACFs that did not use OP was $18.9 \%(1070 / 5672)$ and the attack rate where OP was used was $1.9 \%(810.1017 / \mathrm{S} 09502688120006593 / 4392)$. The absolute risk reduction was $17 \%$ and the number needed to treat to prevent one case was six. Overall, the univariate crude relative risk ratio of $\mathrm{OP}$ failure among ACF residents on $\mathrm{OP}$ was $0.10 \mathrm{RR}$ (95\% CI: 0.08-0.12) $(P<0.0001)$. This indicates that oseltamivir prophylaxis was $90 \%$ effective in preventing new clinical cases of influenza. However, at an ACF level, there was an increased risk of OP failure [6.50 RR (95\% CI: 2.86-14.77)] in facilities with high OP utilization rate.

\subsection{Dementia Wards}

Aged care facilities with dementia wards had 30\% more clinical influenza cases and higher rates of oseltamivir used for treatment (34\%) than for prophylactic purposes $(12 \%)$, in comparison to ACFs without a dementia ward (Figure 1). However, compared with ACFs without dementia wards, ACFs with dementia wards had lower OP failure rates (44\%) indicating increased effectiveness

Table 1 Baseline demographics at an outbreak level

\begin{tabular}{|c|c|c|c|c|c|c|c|c|}
\hline Variable & Outbreaks & Total & Mean & SD & Rate & SD & Min & $\operatorname{Max}$ \\
\hline Total residents & 86 & 10,062 & 117 & 98.1 & & & 14 (Count) & 476 (Count) \\
\hline Laboratory confirmed & 86 & 559 & & & $9 \%$ & $9 \%$ & $0.6 \%$ & $64.3 \%$ \\
\hline Clinical cases (ILI) & 86 & 1152 & & & $16 \%$ & $13 \%$ & $1.4 \%$ & $78.6 \%$ \\
\hline Resident vaccination & 80 & 8280 & & & $88 \%$ & $18 \%$ & $1 \%$ & $100 \%$ \\
\hline Staff vaccination & 58 & 3007 & & & $37 \%$ & $24 \%$ & $0.6 \%$ & $100 \%$ \\
\hline Oseltamivir treatment & 86 & 628 & & & $8 \%$ & $8 \%$ & $0 \%$ & $100 \%$ \\
\hline Oseltamivir prophylaxis & 86 & 4395 & & & $54 \%$ & $33 \%$ & $0 \%$ & $100 \%$ \\
\hline Cases of OP failure & 84 & 84 & & & $1 \%$ & $2 \%$ & $0 \%$ & $10.4 \%$ \\
\hline Hospitalization rate & 86 & 138 & & & $2 \%$ & $2 \%$ & $0 \%$ & $10.2 \%$ \\
\hline Deaths & 86 & 34 & & & $0 \%$ & $0.01 \%$ & $0 \%$ & $0.035 \%$ \\
\hline Number of areas & 86 & 439 & 5.1 & 5.2 & & & & \\
\hline Areas affected & 86 & 146 & 1.7 & 1 & $5 \%$ & $31 \%$ & 1 (Count) & 25 (Count) \\
\hline Outbreak duration after PHU notified (days) & 86 & 560 & 6.5 & 4.7 & & & 0 (Count) & 23 (Count) \\
\hline Days until PHU notified & 86 & 464 & 5.4 & 3.7 & & & 1 (Count) & 19 (Count) \\
\hline Days until precautions & 86 & 464 & 5.4 & 3.7 & & & 1 (Count) & 19 (Count) \\
\hline Variable & Outbreaks & & Low care & Both care & \multicolumn{2}{|c|}{ High care } & & \\
\hline \multirow[t]{2}{*}{ Low-both-high level care } & 86 & & $16(19 \%)$ & $54(63 \%)$ & \multicolumn{2}{|c|}{$16(19 \%)$} & & \\
\hline & & & Yes & No & & & & \\
\hline Dementia ward & 86 & & $70(81 \%)$ & $16(19 \%)$ & & & & \\
\hline
\end{tabular}

ILI, influenza-like illness; OP, Oseltamivir prophylaxis; PHU, Public Health Unit.

$\begin{array}{lr}\text { Outcome } & \text { Relative Risk (95\% Cl) } \\ \text { Clinical Cases } & 1.3(1.11-1.53) \\ \text { Lab Cases } & 1.02(0.82-1.25) \\ \text { Deaths } & 1.38(0.53-3.56) \\ \text { Hospitalisation } & 0.83(0.56-1.24) \\ \text { OP Failure Rate } & 0.56(0.34-0.93) \\ \text { Oseltamivir Treatment Rate } & 1.34(1.08-1.67) \\ \text { Oseltamivir Prophylaxis Rate } & 0.88(0.81-0.94) \\ \text { Staff Vaccination Rate } & 1.68(1.50-1.90) \\ \text { Resident Vaccination Rate } & 1.04(0.98-1.1) \\ \text { Areas Affected } & 1.45(1.1-1.92)\end{array}$

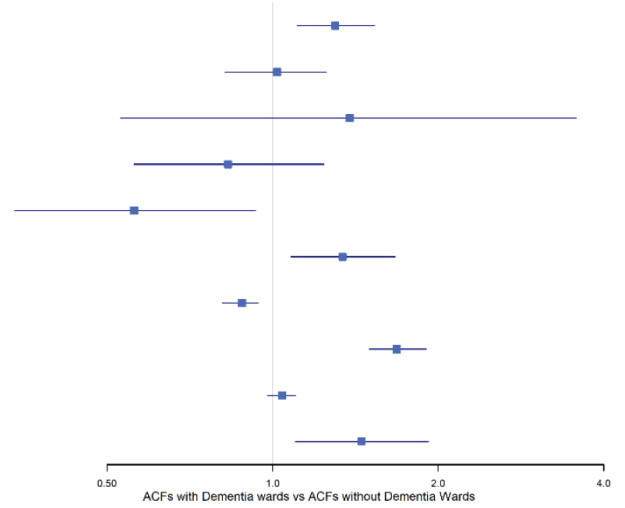

Figure 1 Relative risk of clinical outcomes in influenza outbreaks in dementia wards compared with non-dementia wards in ACFs. ACF, aged care facility; OP, oseltamivir prophylaxis; deaths, any deaths occurring in residents of the ACF during the outbreak. 


$\begin{array}{lr}\text { Outcome } & \text { Relative Risk (95\% Cl) } \\ \text { Clinical Cases } & 0.71(0.58-0.87) \\ \text { Lab Cases } & 0.86(0.66-1.13) \\ \text { Deaths } & 0.56(0.15-2.11) \\ \text { Hospitalisation } & 1.01(0.57-1.78) \\ \text { OP Failure Rate } & 0.13(0.05-0.38) \\ \text { Oseltamivir Treatment Rate } & 1.17(0.88-1.56) \\ \text { Oseltamivir Prophylaxis Rate } & 1.1(0.99-1.22) \\ \text { Staff Vaccination Rate } & 0.82(0.71-0.94) \\ \text { Resident Vaccination Rate } & 0.94(0.87-1.02) \\ \text { Areas Affected } & 1.74(1.30-2.33)\end{array}$

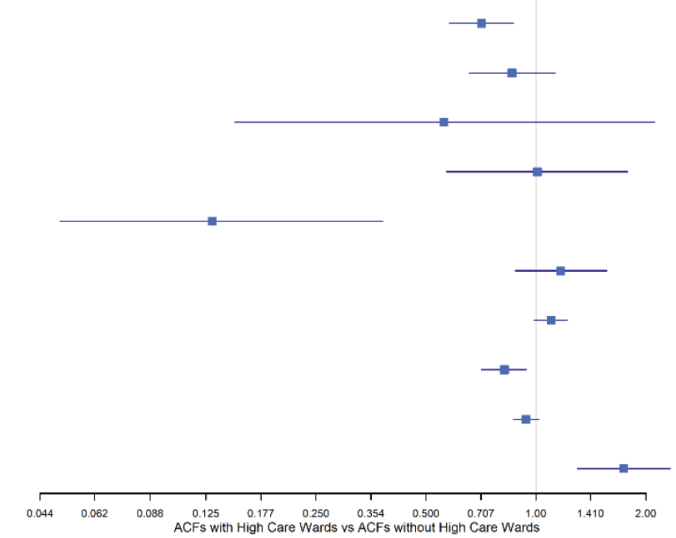

Figure 2 Relative risk of clinical outcomes in influenza outbreaks in high care wards compared with non-high care wards in ACFs. ACF, aged care facility; OP, oseltamivir prophylaxis; deaths, any deaths occurring in residents of the ACF during the outbreak.

of OP in high care settings. ACFs with only high care wards had $29 \%$ fewer clinical cases. Although the rates of oseltamivir prescription were the same in high care only ACFs versus other ACFs, the OP failure rate was $87 \%$ lower in the high care ACFs, indicating oseltamivir was more effective in preventing clinical cases in the high care setting (Figure 2).

\subsection{PHU Notification Delay}

For each day an ACF delayed notifying the PHU of an influenza outbreak there was a $6 \%$ increase in hospitalization [1.06; (95\% CI: $1.02-1.10)$ ] among residents. For each day an ACF delayed notifying the PHU the duration of the outbreak was extended by 0.42 days (95\% CI: 0.16-0.68).

\section{DISCUSSION}

Influenza outbreaks in ACFs cause considerable burden of illness among residents and staff, and have a significant impact on the health system through increased hospitalizations. The lack of appropriate treatment and preventive strategies for influenza in ACFs leads to a greater burden of illness and results in poorer outcomes. Whereas there is variable evidence to support the use of $\mathrm{OP}$ in ACFs [15], this large cohort study adds to the growing body of evidence on the effectiveness of oseltamivir used prophylactically to prevent influenza in ACFs during an outbreak. This study found that when used as prophylaxis, oseltamivir was highly effective in preventing new clinical cases at an individual level in influenza outbreaks in dementia-specific units and high care settings when compared with nondementia-specific wings and low care settings.

Our findings support that of an RCT by Booy et al. [13] comparing treatment dose and treatment plus prophylactic antiviral therapies that found that the use of prophylactic treatment reduced both the attack rate and duration of the outbreak. Our study identified an even greater reduction in the attack rate (90\%) when compared with the RCT (36\%). Our findings, which are from a much larger cohort ( $>10,000$ patients vs 672 patients), provide strong evidence that OP is highly effective in preventing new cases in high care settings and dementia wards. However, OP is often under-used in these settings and oseltamivir for treatment purposes is preferred by prescribers due to the greater availability of evidence to support its use for treatment [16]. Moreover, there is variability in prescribing practice among primary care physicians, and earlier studies on OP in this same location lacked sufficient power to detect differences [11].

This study also found that OP failure was 6.5 (95\% CI: 2.86-14.77) times higher in ACFs that had a higher OP utilization rate, which appears contradictory to the previous statement on the effectiveness of OP to prevent new cases of illness at the individual level. However, this seemingly contradictory finding is evidence of indication bias because the failure rate at the facility level is different from that at the individual level, where the OP failure rate at the ACF level is confounded by the severity of the outbreak. Severe outbreaks are usually characterized by higher attack rates, proportion infected, case-fatality rates, hospital admission rates, and longer outbreak duration [17]. In our study, the indication bias is reflective of the investigations that are carried out at the facility level. Notwithstanding, the study identified a significant reduction in the number of new cases at the individual level, which is noteworthy for public health practice given antivirals are administered clinically at an individual and not at a facility level [18].

Delays in reporting outbreaks to the PHU and in implementation of precautionary measures as well as use of OP in non-influenza illnesses are some of the likely contributing factors of more hospitalizations and longer outbreak duration. The results of our study show that there was a $6 \%$ increase in the hospitalization rate for each day ACFs delayed reporting the outbreak to the PHU. This increase is clinically and statistically significant, and demonstrates that early public health involvement along with OP use has the potential to contain an outbreak promptly. This finding is in keeping with findings from other studies conducted in metropolitan NSW [19]. These results also support the need for mandatory reporting of influenza outbreaks by ACFs to PHUs to prevent unnecessary morbidity and mortality. Current strategies being used by PHUs to identify unreported outbreaks include the linking of the addresses of single notified cases to residential aged care facilities (RACF) locations [20] and an online system (FluCARE), which is currently being piloted in metropolitan Sydney $[21,22]$. 
Despite annual influenza vaccinations for ACF residents and staff being one of the main preventive measures, vaccine efficacy is limited among residents and uptake among staff is low. Strategies for early PHU intervention should include education of ACF staff around interventions such as encouragement of annual influenza vaccinations as a preventive measure and consistently monitoring and identifying residents with influenza-like symptoms. This is especially important as research has indicated that elderly residents can have atypical presentations of influenza, therefore periodic testing for influenza even in the absence of fever may be warranted during the influenza season [23,24]. Active surveillance by PHU staff, such as phone calls to the ACFs during influenza season, is also an important strategy for the early identification of influenza outbreaks and could potentially lead to less morbidity and mortality. Furthermore, strategies to minimize the control of an outbreak such as limiting ill visitors and staff from the facility especially when influenza is known to be circulating within the general community and increasing staff vaccination rates are vital to preventing complications of an outbreak such as hospitalization and mortality.

This study has several limitations. The rate of increasing prescriptions of oseltamivir across the ACF during an outbreak was not standardized in the cohort and this could impact the rate of use for treatment or prophylaxis in individual ACFs. Challenges faced by ACFs to increase OP usage include prescriber preference for using oseltamivir for treatment rather than for prevention, the clinician's perception of the risk-benefit profile, renal adjustment, and cost. The analysis of outbreak-related data in a residential care setting is subject to confounding effects from multiple covariates, several of which are not easily measured or controlled in a cohort study $[25,26]$.

Although this study is conducted on influenza outbreaks in ACFs in South Western Sydney, these results for OP are generalizable to locations that follow similar outbreak management protocols and recommend similar infection control practices. This study provides rationale for pooling outbreak data from a wider cross-section of jurisdictions using similar protocols to provide high-quality evidence to guide the appropriate use of antivirals in ACFs during influenza outbreak responses.

\section{CONCLUSION}

Despite precautionary measures and annual vaccinations, influenza still poses a high risk of morbidity and hospitalization in ACF settings and the use of antiviral prophylaxis is imperative to effective outbreak management.

This study reveals that provision of antiviral prophylaxis during ACF influenza outbreaks is an effective strategy to prevent new cases of influenza especially in ACFs with dementia wards and high care settings. Prompt notification of outbreaks by ACFs to PHUs is crucial to reduce unnecessary hospitalizations and reduce outbreak duration. Such an approach requires collaboration between the ACFs and local PHUs to ensure timely and effective management and must be supported by relevant policy framework. Mandatory reporting of influenza outbreaks to PHUs would ensure that ACFs are supported throughout the outbreak and based on our findings, should facilitate a reduction in hospitalizations and mortality. Better control of influenza among staff, such as through higher vaccination coverage, could lead to better control of outbreaks, which subsequently should contribute to disease control in the wider local community.

\section{CONFLICTS OF INTEREST}

The authors declare they have no conflicts of interest.

\section{AUTHORS' CONTRIBUTION}

SF-L, MD, HL contributed to the conceptualization of the paper. HL carried out data cleaning. MD analyzed the data. SF-L, MD, $\mathrm{HL}, \mathrm{KA}, \mathrm{NP}$, and LB wrote the manuscript. SF-L and NP carried out quality checks and reviewed the manuscript.

\section{FUNDING}

There was no specific funding provided for this study, which was carried out as part of routine public health surveillance activities.

\section{ACKNOWLEDGEMENTS}

The authors would like to acknowledge all Public Health Unit staff who contributed to outbreak surveillance and data collection during the period 2015-2018.

\section{REFERENCES}

[1] Sayers G, Igoe D, Carr M, Cosgrave M, Duffy M, Crowley B, et al. High morbidity and mortality associated with an outbreak of influenza $\mathrm{A}(\mathrm{H} 3 \mathrm{~N} 2)$ in a psycho-geriatric facility. Epidemiol Infect 2013;141;357-65.

[2] New South Wales Government. Disease Reporting Laboratories. Public Health Act 2010; 2019. Available from: https://www.health. nsw.gov.au/Infectious/Documents/ph-labs-notifications.pdf.

[3] Rosewell A, Chiu C, Lindley R, Dwyer DE, Moffatt CRM, Shineberg C, et al. Surveillance for outbreaks of influenza-like illness in the institutionalized elderly. Epidemiol Infect 2010;138;1126-34.

[4] Andrews N, McMenamin J, Durnall H, Ellis J, Lackenby A, Robertson C, et al. Effectiveness of trivalent seasonal influenza vaccine in preventing laboratory-confirmed influenza in primary care in the United Kingdom: 2012/13 end of season results. Euro Surveill 2014;19;5-13.

[5] Talbot HK. Influenza in older adults. Infect Dis Clin N Am 2017;31;757-66.

[6] Ye M, Jacobs A, Khan MN, Jaipaul J, Oda J, Johnson M, et al. Evaluation of the use of oseltamivir prophylaxis in the control of influenza outbreaks in long-term care facilities in Alberta, Canada: a retrospective provincial database analysis. BMJ Open 2016;6;e011686.

[7] Monto AS, Rotthoff J, Teich E, Herlocher ML, Truscon R, Yen HL, et al. Detection and control of influenza outbreaks in well-vaccinated nursing home populations. Clin Infect Dis 2004;39;459-64.

[8] Baum SG. Oseltamivir and the influenza alphabet. Clin Infect Dis $2006 ; 43 ; 445-6$. 
[9] McClellan K, Perry CM. Oseltamivir: a review of its use in influenza. Drugs 2001;61;263-83.

[10] Fiore AE, Fry A, Shay D, Gubareva L, Bresee JS, Uyeki TM, et al. Antiviral agents for the treatment and chemoprophylaxis of influenza - recommendations of the Advisory Committee on Immunization Practices (ACIP). MMWR Recomm Rep 2011;60;1-24.

[11] Merritt T, Hope K, Butler M, Durrheim D, Gupta L, Najjar Z, et al. Effect of antiviral prophylaxis on influenza outbreaks in aged care facilities in three local health districts in New South Wales, Australia, 2014. Western Pac Surveill Response J 2016;7;14-20.

[12] van der Sande MAB, Meijer A, Şen-Kerpiclik F, Enserink R, Cools HJM, Overduin P, et al. Effectiveness of post-exposition prophylaxis with oseltamivir in nursing homes: a randomised controlled trial over four seasons. Emerg Themes Epidemiol 2014;11;13.

[13] Booy R, Lindley RI, Dwyer DE, Yin JK, Heron LG, Moffatt CRM, et al. Treating and preventing influenza in aged care facilities: a cluster randomised controlled trial. PLoS One 2012;7;e46509.

[14] NSW Ministry of Health. Influenza control guideline. Infectious Diseases - Control Guidelines 2018; 2019. Available from: https://www.health.nsw.gov.au/Infectious/controlguideline/ Pages/influenza.aspx.

[15] Gorišek Miksić N, Uršič T, Simonović Z, Lusa L, Lobnik Rojko P, Petrovec M, et al. Oseltamivir prophylaxis in controlling influenza outbreak in nursing homes: a comparison between three different approaches. Infection 2015;43;73-81.

[16] World Health Organization. Prevention and control of outbreaks of seasonal influenza in long-term care facilities: a review of the evidence and best-practice guidance. Geneva: World Health Organization; 2017. Available from: http://www.euro.who.int/_data/assets/pdf_ file/0015/330225/LTCF-best-practice-guidance.pdf?ua=1.

[17] Utsumi M, Makimoto K, Quroshi N, Ashida N. Types of infectious outbreaks and their impact in elderly care facilities: a review of the literature. Age Ageing 2010;39;299-305.
[18] Bosco JL, Silliman RA, Thwin SS, Geiger AM, Buist DS, Prout $\mathrm{MN}$, et al. A most stubborn bias: no adjustment method fully resolves confounding by indication in observational studies. J Clin Epidemiol 2010;63;64-74.

[19] Tennant E, Fletcher S, Kakar S, Najjar Z, Lord H, Clark P, et al. Factors associated with adverse outcomes during influenza outbreaks in aged care facilities. Aust N Z J Public Health 2020;44; 65-72.

[20] Boonwaat L, Fletcher-Lartey S, Conaty S. Underreporting of influenza outbreaks in aged care facilities in South Western Sydney, Australia, 2014. Western Pac Surveill Response J 2016;7;31-3.

[21] Sydney Local Health District. New app to help save lives during flu outbreaks. Sydney connect; Camperdown, New South Wales, Australia. 2019. Available from: https://www.slhd.nsw.gov.au/ sydneyconnect/story-New-app-to-help-save-live.html.

[22] Australian Ageing Agenda. New app helps staff identify flu outbreaks. Australian Ageing Agenda; 2019. Available from: https:// www.australianageingagenda.com.au/noticeboard/resources/ new-app-helps-staff-identify-flu-outbreaks/.

[23] Álvarez-Lerma F, Marín-Corral J, Vila C, Masclans JR, González de Molina FJ, Martín Loeches I, et al. Delay in diagnosis of influenza A (H1N1)pdm09 virus infection in critically ill patients and impact on clinical outcome. Crit Care 2016;20;337.

[24] Haber N, Khelili D, Martineau D, Dekimeche S, Szekely C, Lebon P. Delay in diagnosis of influenza virus in an elderly hospitalized patient: a fatal outcome. Clin Med Insights Case Rep 2012;5;5-8.

[25] Lansbury LE, Brown CS, Nguyen-Van-Tam JS. Influenza in long-term care facilities. Influenza Other Respir Viruses 2017;11;356-66.

[26] Rainwater-Lovett K, Chun K, Lessler J. Influenza outbreak control practices and the effectiveness of interventions in long-term care facilities: a systematic review. Influenza Other Respir Viruses $2014 ; 8 ; 74-82$. 